\title{
Warum und wie die Liberalisierung des Cannabismarktes in Deutschland erfolgen sollte
}

\author{
Der vorliegende Beitrag fasst die Argumente für eine Legalisierung von Cannabis zusammen \\ und macht Vorschläge zur Ausgestaltung des Regulierungsrahmens. Hauptargument für \\ eine regulierte Cannabisfreigabe sind nicht die zu erwartenden Steuereinnahmen oder die \\ entstehenden legalen Arbeitsplätze, sondern der verbesserte Jugend- und Gesundheitsschutz \\ in einem staatlich regulierten Cannabismarkt. Während es bei legalen Suchtmitteln wie \\ Tabak und Alkohol erfolgreich gelingt, den Konsum immer weiter einzudämmen, nimmt \\ der Cannabiskonsum trotz Prohibition seit Jahren zu. Entscheidend für einen effektiven \\ Jugend- und Gesundheitsschutz ist eine wohl durchdachte Marktordnung mit sachgerechter \\ Marktregulierung und Besteuerung.
}

\begin{abstract}
Im Koalitionsvertrag hat die neue Bundesregierung die Legalisierung von Cannabis zu Genusszwecken in Deutschland wie folgt angekündigt: „Wir führen die kontrollierte Abgabe von Cannabis an Erwachsene zu Genusszwecken in lizenzierten Geschäften ein. Dadurch wird die Qualität kontrolliert, die Weitergabe verunreinigter Substanzen verhindert und der Jugendschutz gewährleistet. Das Gesetz evaluieren wir nach vier Jahren auf gesellschaftliche Auswirkungen“ (SPD, Bündnis 90/Die Grünen und FDP, 2021).
\end{abstract}

Damit folgt die Bundesregierung einem internationalen Trend. Schon seit Oktober 2018 ist der Verkauf von Marihuana in Kanada auch zu Genusszwecken legal. Damit war Kanada der erste G7-Staat und überhaupt das erste Industrieland, in dem Anbau, Handel und Konsum von Cannabis nicht nur entkriminalisiert wurden, sondern landesweit auch für Genusszwecke vollständig legal sind. Für medizinische Zwecke sind Anbau und Verkauf von

(C) Der/die Autor:in 2022. Open Access: Dieser Artikel wird unter der Creative Commons Namensnennung 4.0 International Lizenz veröffentlicht (creativecommons.org/licenses/by/4.0/deed.de).

Open Access wird durch die ZBW - Leibniz-Informationszentrum Wirtschaft gefördert.

Prof. Dr. Justus Haucap ist Direktor des Düsseldorf Institute for Competition Economics (DICE) an der Heinrich-Heine-Universität in Düsseldorf.

Leon Knoke ist dort Research Assistant.
Cannabis auf Rezept hingegen schon seit längerer Zeit in vielen Staaten legal und auch vergleichsweise wenig umstritten. Vor der Liberalisierung des Cannabismarktes in Kanada haben bereits eine ganze Reihe von Staaten den Konsum von Cannabis entkriminalisiert. Auch in Deutschland ist der Cannabiskonsum in Folge eines Urteils des Bundesverfassungsgerichts aus dem Jahr 1994 faktisch weitgehend straffrei gestellt. Strafverfahren wegen des Erwerbs von „Kleinstmengen“ an Cannabisprodukten werden in der Regel von der Staatsanwaltschaft eingestellt, wobei die als Kleinstmenge definierte Menge in den Bundesländern unterschiedlich geregelt ist (Wissenschaftliche Dienste des Deutschen Bundestags, 2019a).

Einzelne industriell weniger entwickelte Länder wie vor allem Uruguay sowie einzelne US-Bundestaaten wie Colorado, Washington (jeweils seit 2014) und seit 2018 auch Kalifornien haben auch Anbau, Handel und Konsum von Cannabis in unterschiedlichem Ausmaß liberalisiert. Inzwischen haben in den USA 18 Bundesstaaten sowie Washington D.C. den Konsum von Cannabis zu Genusszwecken legalisiert. Die Legalisierungsmodelle unterscheiden sich dabei aber durchaus.

In Uruguay etwa kontrolliert, vertreibt und verkauft der Staat Cannabis unter anderem über registrierte Apotheken. Das Gesetz zur Regelung des Cannabismarktes hat sequenziell drei Bezugsquellen zugelassen: 2016 zunächst den registrierten Eigenanbau von maximal sechs Pflanzen, 2017 Non-Profit Cannabis-Clubs für maximal 45 Gesellschafter:innen und seit Juli 2018 den Verkauf in Apotheken. Für die letzte Option haben sich mit ihren Fingerabdrücken bis Juli 201813.000 Nutzende registrieren lassen, denn nur Uruguayer:innen dürfen maximal zehn Gramm Cannabis pro Woche kaufen (Olaso, 2018). 
Im Oktober 2020 waren es 40.000 registrierte Nutzende. Jedoch gibt es im ganzen Land nur 14 registrierte Apotheken, die legal Cannabis abgeben (Tagesschau, 2020).

Auch in Deutschland hat die Debatte über Legalisierung versus Prohibition von Cannabis in den vergangenen Jahren an Fahrt aufgenommen. Selbst in eher konservativen Medien wie der Tageszeitung „Die Welt" oder der „Frankfurter Allgemeinen Zeitung" fanden sich vermehrt Plädoyers für eine legalisierte Freigabe von Cannabis (Welt, 2018; FAZ, 2017). Schon 2015 hatte die Wirtschaftswoche gleich auf der Titelseite Ludwig Erhard mit Joint statt Zigarre in der Hand abgebildet und unter dem Titel „Haschisch für alle" ein ökonomisches Plädoyer für die Cannabisfreigabe gedruckt (WiWo, 2015). Selbst in der CDU gab es zumindest einzelne Stimmen für eine Aufgabe oder kritische Überprüfung der bisherigen Prohibitionspolitik (vgl. z.B. Zeit, 2016). Im September 2018 hatte sich auch der damalige Vorsitzende des Gesundheitsausschusses des Deutschen Bundestags, Erwin Rüddel (CDU), zumindest für Cannabismodellprojekte ausgesprochen (DAZ, 2018).

Viele Ökonom:innen, darunter auch mehrere Nobelpreisträger, sprechen sich schon lange für eine kontrollierte Freigabe von Cannabis auch für Konsumzwecke jenseits der medizinischen Nutzung aus (Schomberg, 2014). Dabei konzedieren zahlreiche Befürwortende einer regulierten Freigabe durchaus, dass auch bei einer Legalisierung der „Problemkonsum“ nicht völlig eliminiert wird und somit gesellschaftliche Probleme des Cannabiskonsums nicht völlig verschwinden werden. Jedoch verschwinden diese Probleme - wie die Erfahrungen im In- und Ausland eindrucksvoll belegen - auch bei der bisherigen Prohibitionspolitik eben nicht.

Anders als in der Öffentlichkeit manchmal vermutet wird, treten die meisten Ökonom:innen nicht primär deswegen für die Cannabisfreigabe ein, weil sie kalten Herzens die gesundheitlichen Risiken ignorieren und nur mögliche Steuereinnahmen und (legale) Arbeitsplätze im Auge haben, wenngleich beides positive Nebeneffekte einer Legalisierung wären (Haucap und Knoke, 2021a, 2021b). Ganz im Gegenteil, gerade weil Cannabis - sofern es nicht gerade für medizinische Zwecke eingesetzt wird - gesundheitsgefährdend sein kann, treten viele Ökonom:innen dafür ein, dem organisierten Verbrechen die Kontrolle über den Markt wirksam zu entziehen und stattdessen eine behördliche Kontrolle des Marktes mit strikten Regeln einzuführen.

\section{Ökonomische Gründe für die Regulierung von} Cannabis

Aus ökonomischer Perspektive sind Märkte genau dann regulierungsbedürftig, wenn erstens ohne eine Regulierung
Marktversagen droht und zweitens zugleich die Regulierung mit hinreichender Sicherheit zu besseren Ergebnissen führt (Coase, 1960 sowie Demsetz, 1969). Als klassische Marktversagenstatbestände gelten erhebliche Wettbewerbsprobleme (im Extremfall das natürliche Monopol), negative oder positive externe Effekte (als Spezialfall öffentliche Güter) sowie Informationsdefizite. Verhaltensökonomische Erkenntnisse können zudem auch dann regulatorische Eingriffe rechtfertigen, wenn hinreichend viele Nachfragende (oder gegebenenfalls auch Anbietende) unter verzerrten Wahrnehmungen leiden oder systematisch Entscheidungsfehler begehen („Biases“), welche sich ex post nur schwer korrigieren lassen. Aus politischer Sicht lässt sich ein regulatorischer Eingriff in einer Demokratie zudem rechtfertigen, wenn eine Mehrheit der Bevölkerung eine solche Regulierung wünscht. Gleichwohl besteht in letzterem Fall auch insofern ein Spannungsfeld, als dass regulatorische Eingriffe die Freiheit der Minderheit beschränken können und damit Freiheitsrechte einschränken.

Welches potenzielle Marktversagen rechtfertigt nun einen regulatorischen Eingriff in den Cannabismarkt? Aus klassischer ökonomischer Perspektive kommen vor allem Informationsasymmetrien in Frage, eventuell auch externe Effekte. Informationsasymmetrien können eine Rolle spielen, da Hersteller:innen und Händler:innen illegaler Drogen oftmals besser über die Qualität der Ware informiert sind als die potenzielle Kundschaft. Dies betrifft zum einen den Reinheitsgrad der Suchtmittel und die genauen Inhaltsstoffe. Bei Cannabis etwa variiert der THCGehalt zwischen den verfügbaren Produkten erheblich. Des Weiteren finden sich teilweise äußerst gesundheitsschädliche Streckmittel in Cannabisprodukten ${ }^{1}$ und auch die Belastung mit Pestiziden ist teilweise sehr hoch. Für Nachfragende sind diese Qualitätsaspekte von außen oft nicht leicht zu erkennen. Informationsasymmetrien allein rechtfertigen jedoch keine Prohibition, sondern sprechen eher für Kennzeichnungspflichten, Gütesiegel und gegebenenfalls Qualitätsvorschriften und -kontrollen.

Aufgrund der solidarischen Finanzierung des Gesundheitssystems können durch den Konsum von Suchtmitteln auch externe Effekte in dem Sinne auftreten, dass die gesundheitlichen Folgekosten des Drogenkonsums nicht individuell getragen werden, sondern von der Versichertengemeinschaft, und diese Externalität bei der Konsumentscheidung nicht hinreichend berücksichtigt wird. Gleichwohl spricht auch dieses potenzielle Problem nicht für eine Prohibition, sondern eher für eine gesonderte Besteuerung des Cannabiskonsums, wie sie auch bei Alkohol und Tabak zu finden ist. Andere Externalitäten, wie etwa eine durch Drogenkonsum ausgelöste Beschaf-

1 Vgl. etwa die regelmäßigen Warnungen auf www.dirty-weed.com. 
fungskriminalität, sind bei Cannabis aufgrund der relativ geringen Preise, anders als bei manchen "harten Drogen“, kein wirklich nennenswertes Problem.

Aus der klassischen Marktversagenstheorie lässt sich somit eine Rechtfertigung für die Prohibition von Cannabis nicht ableiten. Neuere verhaltensökonomische Theorien liefern jedoch zusätzliche Rechtfertigungen für staatliche Markteingriffe. So ist durchaus plausibel, dass einige Personen die Folgen ihres Konsums falsch einschätzen, gerade auch was etwaige Suchteffekte angeht. Eine Prohibition wäre in diesem Fall dann gerechtfertigt, wenn (a) hinreichend viele Konsumierende die negativen Konsequenzen des Drogenkonsums unterschätzen, (b) diese negativen Konsequenzen bei hinreichend vielen Nutzenden auch erheblich sind und (c) die Prohibition für alle Konsumierenden von allen verfügbaren Instrumenten und Optionen zur Intervention die adversen Konsequenzen tatsächlich für alle Kundengruppen am besten adressieren oder gar unterbinden kann. Sollte es etwa ausreichend sein, den Konsum für Minderjährige zu untersagen, weil diese die negativen zukünftigen Effekte des Konsums besonders systematisch unterschätzen, so wäre eine Prohibition für Minderjährige ausreichend (mit entsprechenden Strafen für den Verkauf oder die Weitergabe an Minderjährige), eine Prohibition für ältere Konsumentengruppen hingegen nicht notwendig. Im Grunde geht es also um eine paternalistische Begründung für die Prohibition. Ausreichend für die Legitimation der generellen Prohibition ist somit nicht allein der Befund, dass Jugendliche die Konsequenzen des Suchtmittelkonsums stark unterschätzen, dies aber für sie besonders schädlich ist. Dieses Problem könnte nämlich auch durch eine Altersbeschränkung (wie bei Alkohol und Tabak) adressiert werden. Zu zeigen wäre von Befürwortenden der allgemeinen Prohibition hingegen, dass auch ältere Konsumierende die Konsequenzen systematisch falsch einschätzen oder die Weitergabe an Jugendliche nur durch die Prohibition wirksam unterbunden werden kann. Die empirische Evidenz steht jedoch gerade dazu im Widerspruch, wie wir sehen werden.

\section{Die Funktionsweise des Markts für Cannabis}

Trotz Illegalität und trotz fehlender Möglichkeiten, rechtlich bindende Verträge zu schließen, funktioniert der Markt für Cannabis in Deutschland heute faktisch. Anbietende und Nachfragende tauschen täglich Ware gegen Geld. Die Nachfrageseite ist durch eine große Heterogenität gekennzeichnet. Während der Großteil der Nachfragenden lediglich gelegentlich Cannabis konsumiert und somit mengenmäßig nur einen kleinen Teil der Gesamtnachfrage ausmacht, konsumieren relativ wenige Intensivnutzer:innen den mengenmäßig größeren Teil der nachgefragten Menge.
Ganz allgemein gibt es auf illegalen Drogenmärkten wie auf jedem anderen Markt Angebot und Nachfrage. Eine Besonderheit illegaler Märkte im Vergleich zu gewöhnlichen (legalen) Märkten besteht darin, dass Verträge nicht mit staatlicher Gewalt durch Gerichte durchgesetzt werden können. Aus diesem Grund haben sich auf illegalen Drogenmärkten andere Durchsetzungsmechanismen entwickeln, wie z.B. „private“ Gewalt. Der Drogenhandel basiert zu großen Teilen auf Vertrauen, nicht selten vor dem Hintergrund von Gewaltandrohungen zwischen Händlern und Großhändlern.

Die Angebotsseite zeichnet sich erstens dadurch aus, dass Anbietende primär an Profiten und besonders am Vertrieb margenstarker Produkte interessiert sind. Die Gewinne im Drogenmarkt sind insgesamt (inklusive Großhandel) aufgrund von Monopol- und Kartellbildung typischerweise hoch und ziehen Konkurrenz an (Lopez, 2014). Dieser Wettbewerb findet jedoch anders als auf legalen Märkten nicht über Preise und Qualitäten statt, sondern wird nicht selten mit Gewalt ausgeführt. Die hohen Gewinnaussichten bieten zum einen starke Anreize zu Innovationen (z. B. neuartige Drogen wie Legal Highs oder neue Vertriebsformen über digitale Kanäle) aber auch zu Betrug, beispielsweise in der Form, dass Substanzen mit "Streckmitteln“ bearbeitet werden, die nicht selten gesundheitsschädlich oder gar giftig sind. Zwischen Anbietenden und Nachfragenden bestehen meist erhebliche Informationsprobleme, was die Qualität der Drogen betrifft. Konsument:innen können die Qualität ex ante nur schwer erkennen und Anbieter:innen wissen in der Regel mehr über die Qualität ihrer Ware als die Nachfrageseite. Hieraus resultieren teilweise erhebliche Qualitätsprobleme, die mit hohen Gesundheitsrisiken einhergehen können. Haftungs- und Informationspflichten auf der Angebotsseite gibt es auf dem Schwarzmarkt naturgemäß nicht. Zwar können teilweise Reputationseffekte und Mund-zuMund-Propaganda von Bedeutung sein, wodurch zumindest eine gewisse Qualitätskontrolle ermöglicht wird. Dies betrifft jedoch insbesondere den Teil des Schwarzmarkts, auf dem die Beteiligten regelmäßig aufeinandertreffen. Für das einmalige Aufeinandertreffen spielen derartige Mechanismen eine nur sehr untergeordnete Bedeutung. ${ }^{2}$ Ein wirksamer Verbraucher- und Jugendschutz ist unter einer Prohibition somit faktisch kaum zu gewährleisten.

Zweitens haben Drogendealer:innen oftmals keine hohen Opportunitätskosten, d. h. sie haben keine guten Alternativen anderweitig Geld zu verdienen, mit der Folge, dass sie meist relativ skrupellos sind und auch bereit sind,

2 Teilweise wird bei Cannabis mittlerweile auch eine gewisse Qualitätskontrolle über Internetplattformen vorgenommen, wie z.B. auf der Seite www.graspreis.de, auf der Preise und Qualität unterschiedlicher Regionen in Deutschland bewertet werden. 
hohe rechtliche Risiken einzugehen (Levitt und Dubner, 2009). Und drittens ist besonders für den Cannabismarkt relevant, dass Cannabis - allein schon aufgrund der Möglichkeit relativ einfach selbst Hanf anzubauen und der daher hohen Wettbewerbsintensität - ein relativ margenschwaches Produkt ist, da Dealer:innen letztlich auch gegen den Eigenanbau der Nutzenden und ihres Bekanntenkreises konkurrieren. Daher haben Dealer:innen prinzipiell einen Anreiz, ihre Kundschaft zum Konsum margenstarker Drogen zu motivieren. Dies sind oftmals „harte Drogen“, die deutlich schwieriger selbst angebaut oder hergestellt werden können.

Die Nachfrageseite zeichnet sich, wie schon erwähnt, durch eine relativ starke Heterogenität aus. Nachfragende sind typischerweise (je nach Droge) relativ preisunsensibel, d.h. sie reagieren kaum auf Preiserhöhungen oder Preissenkungen.

\section{Daten zum Cannabiskonsum in Deutschland}

Cannabis ist die mit Abstand am meisten konsumierte illegale Droge in Deutschland. Dies zeigt sich an sogenannten Prävalenzdaten, die auf repräsentativen Surveys darüber beruhen, wie viel Prozent der Bevölkerung innerhalb einer bestimmten Altersgruppe eine bestimmte Droge mindestens einmal in den vergangenen 30 Tagen (30-Tages-Prävalenz), mindestens einmal in den vergangenen zwölf Monaten (12-Monats-Prävalenz) oder mindestens einmal im ganzen Leben (Lebenszeit-Prävalenz) konsumiert haben. Mit einer Lebenszeit-Prävalenz von $31,9 \%$ und einer $12-$ Monats-Prävalenz von $8,3 \%$ bei den 18- bis 59-Jährigen liegt Cannabis deutlich vor dem Konsum anderer Drogen wie z. B. Kokain, Amphetamine oder MDMA, deren Lebenszeitprävalenzen alle unter $5 \%$ liegen und deren 12-Monats-Prävalenzen bei maximal $1 \%$ oder darunter liegen (Seitz et al., 2019).

Fast jede dritte Person in Deutschland zwischen 18 und 59 Jahren hat somit im Laufe ihres Lebens schon mindestens einmal Cannabis konsumiert, während andere illegale Drogen nicht einmal von jedem Zwanzigsten konsumiert wurden. Mehr als $8 \%$ der deutschen Bevölkerung zwischen 18 und 59 Jahren haben sogar mindestens einmal im vergangenen Jahr Cannabis konsumiert, wohingegen "harte Drogen“ von maximal $1 \%$ der Bevölkerung zwischen 18 und 59 Jahren mindestens einmal in den vergangenen zwölf Monaten konsumiert wurden.

Aus der 12-Monats- und 30-Tages-Prävalenz lassen sich Rückschlüsse ziehen, wie regelmäßig Cannabis von den Befragten konsumiert wird. Von 1995 bis 2018 gibt es einen klar zunehmenden Trend im Cannabiskonsum, was sich insbesondere an der Lebenszeitprävalenz zeigt. Die
Abbildung 1

12-Monats-Prävalenz von Cannabis in verschiedenen Altersgruppen

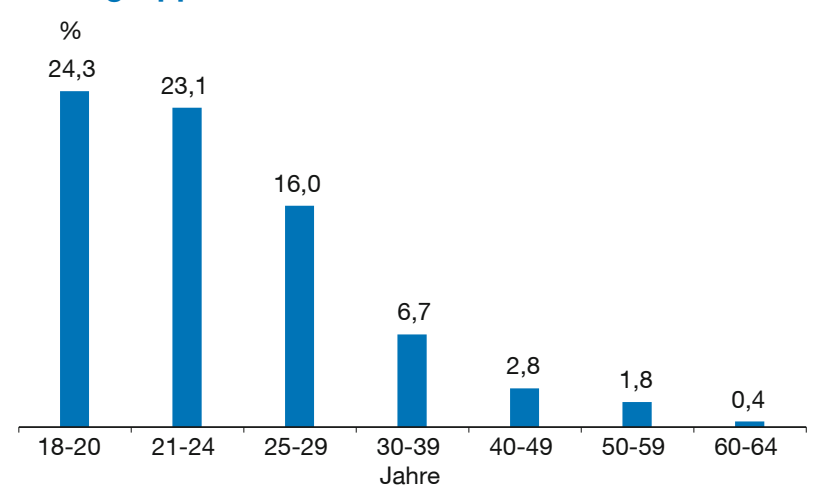

Quelle: Epidemiological Survey of Substance Abuse; eigene Darstellung.

Lebenszeitprävalenz hat sich seit 1995 von 11,9\% nahezu verdreifacht und lag 2018 bei $31,9 \%$. Der Cannabiskonsum hat sich in den vergangenen 25 Jahren deutlich ausgebreitet. Der Trend zeigt sich auch bei der 12-Monats-Prävalenz. 1995 gaben nur 4,4\% der Befragten an, in den vergangenen zwölf Monaten Cannabis konsumiert zu haben und damit knapp 40\% weniger als 2018 mit 8,3\%. Dies lässt darauf schließen, dass nicht nur die Zahl der Konsumierenden gewachsen ist, sondern auch mehr Menschen regelmäßig zu Cannabis greifen. Eine zwischenzeitliche Umkehr lässt sich nur im Zeitraum von 2003 bis 2006 beobachten. Zusammengefasst geben diese Daten Grund zur Annahme, dass nicht nur die Zahl derjenigen, die bereits Cannabis konsumiert haben, angestiegen ist, sondern auch mehr Menschen Cannabis regelmäßig konsumieren.

Cannabis ist dabei offenbar vor allem in jüngeren Altersgruppen attraktiv. Mehr als $40 \%$ der Befragten unter 40 hat bereits mindestens einmal im Leben Cannabis probiert. Etwa jeder Vierte (24,3\%) der Befragten unter 25 gab an, sogar innerhalb der vergangenen zwölf Monate Cannabis konsumiert zu haben, wie Abbildung 1 zeigt (Seitz et al., 2019).

Ein weiteres Indiz für Größe und Wachstum des Cannabismarkts ist die Entwicklung der cannabisbezogenen Rauschgiftdelikte. Von insgesamt 131.951 Fällen im Jahr 2011 über 183.015 Fälle im Jahr 2016 ist diese Zahl auf 220.414 im Jahr 2020 gestiegen, insgesamt ein Anstieg von $67 \%$ (Bundeskriminalamt, diverse Jahrgänge). Das ist ein jährliches Wachstum von etwa $6 \%$. Zu einer Eindämmung des Cannabiskonsums hat die Prohibition offenbar nicht geführt. Im Gegensatz dazu ist der Konsum von Zigaretten in Deutschland pro Kopf im selben Zeitraum um fast $20 \%$ gesunken (Statista, 2021a), der Alkoholkonsum in Deutschland ist auch bei jungen Menschen seit Jahren ebenfalls tendenziell rückläufig (Statista, 
2021b). ${ }^{3}$ Während also der Verbrauch von legalen Suchtmitteln wie Tabak und Alkohol abnimmt, ist bei Cannabis ein stetiger Zuwachs zu verzeichnen. Die Prohibition von Cannabis erreicht somit das gesteckte Ziel der Eindämmung des Konsums offenbar nicht.

Für die Beschreibung des Marktes ist auch der legale Handel mit Cannabis für die medizinische Nutzung von Bedeutung. Im Mai 2011 hat die Legislative die Möglichkeit geschaffen, cannabishaltige Medikamente zuzulassen. Im Juli 2011 ist in Deutschland das erste cannabinoidhaltige Medikament „Sativex" auf den Markt gekommen. Seit März 2017 können Cannabisblüten und Cannabisextrakt in pharmazeutischer Qualität auf einem Betäubungsmittelrezept verschrieben werden. Während im ersten Jahr gut 70.000 cannabishaltige Zubereitungen oder unverarbeitete Cannabisblüten auf ärztliche Verordnung in Apotheken abgegeben wurden (ABDA, 2018), wurden 2020 rund 320.000 Verordnungen im Gegenwert von 150 Mio. Euro bewilligt (Handelsblatt, 2021). In Deutschland bauen inzwischen drei Unternehmen Cannabispflanzen im Auftrag der beim Bundesinstitut für Arzneimittel und Medizinprodukte angesiedelten Cannabisagentur an. Die deutsche Anbaumenge von insgesamt 10,4 Tonnen medizinischem Cannabis ist auf vier Jahre mit je 2,6 Tonnen verteilt und wird zum Preis von 4,30 Euro pro Gramm an die Apotheken verkauft (Tagesschau, 2021). Auch weil bürokratische Hürden eine Verordnung für die Ärzteschaft sehr unattraktiv machen, hat sich der Markt für medizinisches Cannabis nicht so schnell entwickelt wie einige es vor fünf Jahren erwartet haben (Handelsblatt, 2021).

Ökonomische Argumente für eine regulierte Liberalisierung des Cannabismarkts

Das Hauptargument der meisten Ökonom:innen für eine (regulierte) Freigabe von Cannabis liegt - und hier besteht Einigkeit mit vielen Strafrechtler:innen und Suchtforschenden - darin, dass eine kontrollierte Freigabe insbesondere problematische Nutzende besser schützt als die Prohibition, so paradox es sich anhören mag. Faktisch ist Cannabis heute ziemlich problemlos vielerorts zu bekommen, auch und gerade für Jugendliche. Der Konsum von Cannabisprodukten ist unter der bisherigen Prohibitionspolitik nicht merklich gesunken. Dies gilt insbesondere für den „Problemkonsum“ bei Jugendlichen und bei Intensivnutzenden (Seitz et al., 2019).

Illegale Dealer:innen haben weder ein ausgeprägtes Interesse am Jugendschutz noch an einer Qualitätssicherung im Sinne eines Gesundheitsschutzes. Vielmehr

3 Vgl. hierzu auch https://www.kenn-dein-limit.de/alkoholkonsum/alkoholkonsum-in-deutschland/. werden immer wieder äußerst gesundheitsschädliche Substanzen beigemischt ${ }^{4}$, von der Pestizidbelastung der Cannabispflanzen ganz abgesehen. Staatliche Qualitätsvorgaben und -kontrollen finden naturgemäß auf dem Schwarzmarkt nicht statt.

Zugleich haben Dealer:innen tendenziell ein Interesse, ihrer Kundschaft auch härtere Drogen zu verkaufen, da diese regelmäßig eine höhere Profitmarge aufweisen als Cannabis, da Cannabis bzw. Hanf auch leicht selbst angebaut werden kann. Während es nachfrageseitig zumindest umstritten ist, ob Cannabis die Funktion einer Einstiegsdroge hat, gibt es angebotsseitig durchaus einen Zusammenhang, da ein Teil der Dealenden als „Multiproduktanbieter:in“ auftritt und somit ein Interesse hat, Nutzende zum möglichst dauerhaften Konsum der margenstärksten Drogen zu bewegen. Die Profite aus dem Drogengeschäft finanzieren wiederum die Tätigkeiten von Rocker:innenbanden, Mafia und Terrororganisationen.

Eine Legalisierung und kontrollierte Freigabe kann den Schwarzmarkt deutlich reduzieren. Wird Cannabis legalisiert, wird die Nachfrage zumindest in großen Teilen lieber in legalen Geschäften befriedigt als auf dem Schwarzmarkt, solange das legale Cannabis (a) hinreichend verfügbar und (b) nicht zu teuer ist, denn bei legalen Händler:innen kann die Kundschaft von einer gesicherten Qualität ausgehen. Erfahrungen aus Colorado zeigen, dass für legales, kontrolliertes Cannabis durchaus Preise verlangt werden können, die $10 \%$ bis $20 \%$ über den Schwarzmarktpreisen liegen. Letztlich verhält es sich genau wie beim Alkohol. Trotz Alkoholsteuer kauft der ganz große Teil der Bevölkerung den Alkohol aus legalen Quellen und nicht aus Schwarzbrennereien.

Legale Cannabishändler:innen sollten sich jedoch einem Lizenzierungsprozess unterziehen, sodass die Lizenz auch entzogen werden kann, wenn etwa Jugend- und Verbraucherschutzbestimmungen nicht eingehalten werden. Legale Cannabishändler:innen dürften ebenso geringe Anreize haben, ihre Lizenz zu riskieren, indem sie der Kundschaft parallel auch härtere illegale Drogen anbieten, wie heute Wein- oder Spirituosenhändler:innen typischerweise auch nicht parallel illegale Produkte anbieten. Das Problem des „Anfixens“ würde so erheblich reduziert.

Natürlich wird der problematische Cannabiskonsum nicht völlig verschwinden und auch das Problem, dass schon Jugendliche heute Cannabis konsumieren, wäre nicht völlig behoben, ebenso wie auch heute Jugendliche faktisch doch an Spirituosen gelangen (etwa weil volljährige Bekannte die Einkäufe vornehmen). Gleichwohl würde der

4 Vgl. etwa die regelmäßigen Warnhinweise auf www.dirty-weed.com. 
illegale Markt sehr deutlich schrumpfen und das Cannabisgeschäft somit für Dealer:innen und die organisierte Kriminalität weitgehend uninteressant, da das illegale Cannabisgeschäft deutlich weniger profitabel wird, wenn legale, qualitätsgesicherte Markenprodukte auf dem Markt erhältlich sind. Zudem sollte nicht nur die gewerbliche, sondern auch die private Weitergabe von Cannabis an Jugendliche in einem regulierten Markt mit Bußgeldern oder Strafen klar sanktioniert werden.

Der Cannabisverkauf sollte zudem mit verpflichtenden Hinweisen auf gesundheitliche Gefahren und Beratungsangebote für Problemkonsumierende verbunden werden. Im heute illegalen Markt ist es hingegen viel schwieriger die Nutzenden direkt zu adressieren. Auch für die gezielte Aufklärung wäre eine kontrollierte Freigabe daher besser.

Letztlich sprechen auch finanzielle Aspekte für eine kontrollierte Freigabe. Erstens könnten so erhebliche Steuereinnahmen generiert werden, zum einen durch die direkte Besteuerung von Cannabisprodukten, zum anderen durch Gewerbe-, Körperschafts-, Lohn- und Einkommenssteuern im Bereich Cannabisanbau und -handel. Und zweitens würden auch Polizei, Staatsanwaltschaft und Justiz erheblich entlastet, denn durch die Verfolgung von Bagatelldelikten im Zusammenanhang mit Cannabis, bei denen die Verfahren letztlich eingestellt werden, entstehen heute unnötige Kosten. Diese Ressourcen könnten bei Polizei und Staatsanwaltschaft im Falle einer Freigabe sinnvoller eingesetzt werden. Wie wir an anderer Stelle dargelegt haben, gehen wir von einer Summe von 4,7 Mrd. Euro pro Jahr an Steuereinnahmen und Kosteneinsparungen aus (für eine Zusammenfassung vgl. Haucap und Knoke, 2021a). Diese Einnahmen könnten unter anderem für präventive und kurative Maßnahmen eingesetzt werden.

Gegen die Freigabe spricht letztlich nur die Befürchtung, dass bei einer Legalisierung der gesundheitsschädliche Konsum zunehmen könnte. Die Evidenz aus Staaten mit einer liberalen Drogenpolitik wie etwa Portugal oder den USA ist jedoch ermutigend. Zwar ist teilweise durchaus eine Zunahme des Konsums messbar, jedoch geschieht das auch in Staaten, die weiter an der Prohibition festhalten. Differenzen-in-Differenzen-Analysen, welche die Wachstumsraten in Staaten mit legalem Konsum mit solchen Staaten vergleichen, die weiter an der Prohibition festhalten, ergeben keine belastbare Evidenz, dass die Legalisierung kausal für die Zunahme des Cannabiskonsums ist, wie der wissenschaftliche Dienst des Deutschen Bundestags (2019b) dies in einer Zusammenfassung der Studienlage festgehalten hat. Dills et al. (2021) weisen darauf hin, dass der steigende Cannabiskonsum eher als Ursache für die Legalisierung anzusehen ist und nicht als Folge der Cannabisfreigabe.
Interessanterweise scheint gerade der Problemkonsum von Jugendlichen nach einer Legalisierung tendenziell eher ab- als zuzunehmen, zumindest aber scheint er nicht anzusteigen (Washington Post, 2017 sowie Coley et al., 2021). Die Ursachen sind für diese Beobachtung sind noch unklar. Erstens ist denkbar, dass Cannabiskonsum für einige Jugendliche weniger interessant ist, wenn dieser für Erwachsene legal ist. Zweitens ist aber auch zu erwarten, dass es nach einer Legalisierung und dem Schrumpfen des Schwarzmarkts weniger illegale Dealer:innen gibt und somit die Verfügbarkeit von Cannabis für Jugendliche nach der Legalisierung sogar abnimmt, insbesondere wenn komplementär zur Legalisierung für Volljährige die Weitergabe an Jugendliche stärker als bisher bestraft wird und dies mehr ins Zentrum der Strafverfolgung rückt.

Gleichwohl finden Cerda et al. (2020), dass die Prävalenz von Cannabissuchterkrankungen bei Teenagern zwischen 12 und 17 Jahren von 2,18\% auf 2,72\% zugenommen habe, nachdem Colorado, Washington, Alaska und Oregon die Entscheidung zur Cannabislegalisierung gefällt hatten. Unklar bleibt jedoch, warum dies geschieht. Die Autor:innen konzedieren ihren eigenen empirischen Befund, dass der Cannabiskonsum in dieser Altersgruppe gar nicht zugenommen habe (Cerda et al., 2020, 167). Zudem treten die erhöhten Zahlen an Cannabissuchterkrankungen direkt nach der politischen Entscheidung zur Legalisierung auf und damit bereits deutlich vor der faktischen Cannabisfreigabe. Es bleibt leider unklar, warum es schon vor der eigentlichen Freigabe direkt nach der Legalisierungsentscheidung zu einem (geringfügigen) Anstieg an Cannabissuchterkrankungen zu kommen scheint, obwohl der Cannabiskonsum gar nicht steigt.

Selbst bei einer möglichen Zunahme des Cannabiskonsums wäre jedoch dagegen abzuwägen, dass - wie schon erwähnt - im regulierten Markt von einer besseren Qualitätskontrolle auszugehen ist, die verhindert, dass andere gesundheitsschädliche Substanzen beigemischt werden. Sofern sich also Jugendliche illegal Cannabis im legalen Mart besorgen, werden wenigstens die Gesundheitsschäden durch toxische Streckmittel zurückgehen. Gerade aus gesundheitspolitischer Sicht spricht also vieles für eine kontrollierte Freigabe.

\section{Konsequenzen für die Regulierung und Cannabismarktordnung}

Hauptargument für die Legalisierung von Cannabis sind aus ökonomischer Sicht weder die möglichen Steuereinnahmen noch die Arbeitsplätze. Das wichtigste Argument ist vielmehr der verbesserte Jugend- und Gesundheitsschutz in einem legalen Markt. Bei der Ausgestaltung einer Marktordnung, die wirksamen Jugend- und Verbrau- 
cherschutz gewährleistet und den Schwarzmarkt möglichst schnell austrocknet, sollten daher unter anderem folgende Punkte beachtet werden:

Erstens sollte die Legalisierung mit umfassender Information und Aufklärung über die gesundheitlichen Gefahren des Cannabiskonsums einhergehen. Es darf nicht der Eindruck entstehen, der Konsum werde legalisiert, weil Cannabis ungefährlich sei.

Zweitens sollte die gesamte Wertschöpfungskette von Anbau über Verarbeitung bis zum Handel behördlich lizenziert werden. Lizenzverstöße müssen geahndet werden und gegebenenfalls auch mit einem Lizenzentzug sanktioniert werden. Eine flächendeckende Infrastruktur der zuständigen Behörden zur Vergabe von Lizenzen sowie der dazugehörigen Kontrollen der Lizenzen sollte zeitnah aufgebaut werden. Wir schlagen hier ein sich selbst finanzierendes System vor, sodass die Kontrollkosten durch Einnahmen aus Lizenzgebühren gedeckt werden.

Drittens sollte der Vertrieb zum einen über lizenzierte Fachgeschäfte erfolgen. Diese Geschäfte sollten ausschließlich Cannabisprodukte vertreiben (und nicht etwa auch Bier und Chips), sie sollten von Minderjährigen nicht betreten werden dürfen (ähnlich wie Spielhallen), und sie müssen ein Informationsangebot über die gesundheitlichen Gefahren bereithalten. Zum anderen sollte auch Apotheken der Verkauf gestattet werden. Letzteres ist gerade für Gegenden relevant, in denen sich der Betrieb eigener Cannabis-Shops nicht rentieren mag. Wie internationale Erfahrungen zeigen, gelingt es umso schlechter, den Schwarzmarkt auszutrocknen, je weniger lizenzierte Verkaufsstellen es gibt. Zudem sollte sich der Kauf von Cannabis für Erwachsene nicht zu bürokratisch gestalten, da sich zu viele Konsumierende ansonsten Cannabis weiterhin auf dem Schwarzmarkt beschaffen.

Viertens sollte Cannabis besteuert werden. Die Steuer darf jedoch nicht zu hoch sein. Eine Cannabissteuer von 4 Euro pro Gramm wäre ein guter Start, damit Cannabis einerseits nicht zu günstig wird, andererseits aber auch nicht zu teuer (Haucap und Knoke, 2021b, 34-36). Ist Cannabis im legalen Handel zu teuer, wird es nicht gelingen, den Schwarzmarkt auszutrocknen. Neben Grasblüten sollte für andere Produktsorten wie z.B. Edibles analysiert werden, ob hier gegebenenfalls eine Wertsteuer oder eine Mischform einer Mengen- und Wertsteuer sinnvoll wäre. Es ist ebenfalls möglich, den THC-Gehalt zu berücksichtigen, etwa indem Cannabisprodukte je nach inrem THC-Gehalt in zwei oder drei Gruppen eingeteilt werden, die dann unterschiedlich besteuert werden, wie etwa in Illinois und wie dies die FDP vorgeschlagen hatte. So könnten Konsumierende zu einem Kauf von Cannabis mit geringerem THC-Gehalt motiviert werden.
Fünftens sollte es eine Obergrenze für den Eigenanbau geben. In Colorado etwa sind sechs Pflanzen pro Person gestattet. Ohne Beschränkung kann ansonsten zu leicht ein unlizenziertes gewerbliches Angebot entstehen.

Sechstens sollte die Weitergabe von Cannabis an Minderjährige stärker als bisher unter Strafe gestellt werden, um insbesondere den Cannabiskonsum von Teenagern einzudämmen, so wie dies einigen US-Bundesstaaten gelungen ist. Zugleich sollten Strafen bezüglich des Kaufs und Verkaufs von illegalem Cannabis für alle Altersgruppen erhöht werden. Des Weiteren sollten Strafen für Cannabishandelsdelikte erhöht werden, damit Drogendealer:innen trotz geringer Opportunitätskosten stärker abgeschreckt werden.

Siebtens sollte bereits frühzeitig eine Infrastruktur zur Evaluierung der Legalisierungseffekte aufgebaut werden. Es ist zwar zu begrüßen, dass die Bundesregierung vorschlägt, das Gesetz nach vier Jahren auf gesellschaftliche Auswirkungen zu evaluieren. Jedoch sollten beispielsweise die Epidemiologischen Suchtsurveys jährlich statt wie bisher im Drei-Jahres-Rhythmus durchgeführt werden sowie eine Bestandsverfolgung zur Ermittlung des Gesamtbedarfs in Betracht gezogen werden. Zudem sollten Cannabisdaten vergleichbar zum "Cannabis Stats Hub" in Kanada veröffentlicht werden. ${ }^{5}$

Achtens sollten Werberegeln für die Cannabiswirtschaft erlassen werden. Ob ein vollständiges Werbeverbot oder „lediglich“ bestimmte Werbeeinschränkungen (wie etwa bei Tabak) besser geeignet sind, den Problemkonsum zum einen einzudämmen, zum anderen aber auch in legale Bahnen zu lenken, ist bisher offen und nicht zuverlässig auf Basis der verfügbaren Evidenz zu beantworten.

Mit einer solchen Marktordnung sollte es insgesamt jedoch gelingen, die negativen Konsequenzen des Cannabiskonsums deutlich einzudämmen.

\section{Fazit}

Insgesamt gibt es nur wenig Argumente, die gegen eine Cannabisfreigabe in einem regulierten Umfeld sprechen. Die Hoffnung, dass Prohibition und Repression helfen, das Problem zu lösen, hat sich inzwischen deutlich zerschlagen. Im Gegenteil: Die Prohibition ist auf ganzer Linie gescheitert, es ist nicht gelungen den Konsum merklich einzudämmen. Gerade bei Jugendlichen nimmt der Konsum stetig zu. Im Gegensatz dazu ist der Konsum legaler Suchtmittel wie Alkohol und Tabak bei Jugendlichen seit Jahren rückläufig (Statista, 2019, 2021b). Im Klartext heißt dies: Während es bei legalen Suchtmitteln erfolgreich ge-

5 Vgl. hierzu https://www150.statcan.gc.ca/n1/en/catalogue/13-610-X. 
lingt, den Konsum zurückzuführen, ist bei Cannabis trotz Prohibition der gegenläufige Trend zu beobachten. Es ist schwer nachvollziehbar, wie die Cannabisprohibition auf Basis dieser Faktenlage als Erfolg betrachtet werden kann. Einziger Profiteur der repressiven Drogenpolitik bei Cannabis ist heute die organisierte Kriminalität. Die von der Bundesregierung geplante Legalisierung von Cannabis und eine liberalere Drogenpolitik sind nicht nur zu begrüßen, auf Grundlage der vorhandenen Evidenz ist ein solcher Schritt längst überfällig.

\section{Literatur}

ABDA - Bundesvereinigung Deutscher Apothekerverbände (2018), Cannabis: Mehr Abgaben auf Rezept, https://www.abda.de/aktuellesund-presse/newsroom/detail/cannabis-mehr-abgaben-auf-rezept (15. Dezember 2021).

Bundeskriminalamt (diverse Jahrgänge), Bundeslagebild Rauschgiftkriminalität, 2019: 217.929

Cerdá, M., Mauro, C., Hamilton, A., Levy, N. S., Santaella-Tenorio, J., Hasin, D., Wall, M.M., Keyes, K.M. und S.S. Martins (2020), Association Between Recreational Marijuana Legalization in the United States and Changes in Marijuana Use and Cannabis Use Disorder from 2008 to 2016, JAMA Psychiatry, 77(2), 165-171.

Coase, R. H. (1960), The Problem of Social Cost, Journal of Law and Economics, 3, 1-44.

Coley, R. L., C. Kruzik, M. Ghiani, N. Carey, S. S. Hawkins und C. F Baum (2021), Recreational Marijuana Legalization and Adolescent Use of Marijuana, Tobacco, and Alcohol, Journal of Adolescent Health, 69, 41-49.

DAZ - Deutsche Apotheker Zeitung (2018), Öffnet sich die Union in Sachen Cannabis-Legalisierung?, https://www.deutsche-apothekerzeitung.de/news/artikel/2018/09/12/cdu-gesundheitspolitiker-befuerwortet-cannabis-modellversuch-mit-apotheken (15. Dezember 2021).

Demsetz, H. (1969), Information and Efficiency: Another Viewpoint, Journal of Law and Economics, 12(1), 1-22.

Dills, A., S. Goffard, J. Miron und E. Partin (2021), The Effect of State Marijuana Legalizations: 2021 Update, https://www.cato.org/policyanalysis/effect-state-marijuana-legalizations-2021-update\# (15. Dezember 2021).

FAZ (2017), Kiffen erlaubt?, https://www.faz.net/aktuell/wirtschaft/legalisierung-von-cannabis-fdp-und-gruene-fuer-freigabe-15267936.html (15. Dezember 2021).

Handelsblatt (2021), Zahl der Cannabis-Patienten steigt - aber nicht so schnell wie erwartet, https://www.handelsblatt.com/unternehmen/ industrie/marihuana-als-medizin-zahl-der-cannabis-patientensteigt-aber-nicht-so-schnell-wie-erwartet/26793480.html?ticket=ST1366921-x4cweV5b2jWly6cgXQeu-cas01.example.org (15. Dezember 2021).

Haucap, J. und L. Knoke (2021a), Fiskalische Auswirkungen einer Cannabis-Legalisierung in Deutschland, Wirtschaftsdienst, 101(12), 984-986, https://www.wirtschaftsdienst.eu/inhalt/jahr/2021/heft/12/beitrag/fiskalische-effekte-der-cannabis-legalisierung.html (15. Dezember 2021).

Haucap, J. und L. Knoke (2021b), Fiskalische Auswirkungen einer Cannabislegalisierung in Deutschland: Ein Update, https://ideas.repec. org/p/zbw/diceop/112.html (24. Dezember 2021).
Levitt, S. D. und S. J. Dubner (2009), Freakonomics, Penguin Books.

Lopez, G. (2014), The ridiculous profitability of illegal drugs, in one chart, https://www.vox.com/xpress/2014/11/10/7175859/marijuana-legalization-heroin-cocaine-crops (15. Dezember 2021).

Olaso, F. (2018), Marihuana mit Gütesiegel, Deutschlandfunk Kultur, https://www.deutschlandfunkkultur.de/cannabis-paradies-uruguaymarihuana-mit-guetesiegel.1076.de.html?dram:article_id $=422120$ (15. Dezember 2021).

Schomberg, W. (2014), Nobel economists, others urge end to ,war on drugs', Reuters, 6. Mai.

Seitz, N.-N., L. Böttcher, J. Atzendorf, C. Rauschert und L. Kraus (2019), Kurzbericht Epidemiologischer Suchtsurvey 2018 Tabellenband: Trends der Prävalenz des Konsums illegaler Drogen und Drogenmissbrauch und -abhängigkeit nach Geschlecht und Alter 1990-2018, https://www.esa-survey.de/fileadmin/user_upload/Literatur/Berichte/ ESA_2018_Trends_Drogen.pdf (15. Dezember 2021).

SPD, Bündnis 90/Die Grünen und FDP (2021), Koalitionsvertrag 20212025 zwischen SPD, Bündnis 90/Die Grünen und FDP, Mehr Fortschritt wagen.

Statista (2019), Entwicklung des Raucher- und des Nieraucheranteils unter deutschen Jugendlichen im Zeitraum von 1979 bis 2018, https:// de.statista.com/statistik/daten/studie/222992/umfrage/entwicklungdes-raucheranteils-unter-jugendlichen-in-deutschland/ (15. Dezember 2021).

Statista (2021a), Pro-Kopf-Verbrauch von Zigaretten in Deutschland in den Jahren 1964 bis 2020, https://de.statista.com/statistik/daten/studie/6209/umfrage/entwicklung-des-zigarettenkonsums-seit-1964/ (15. Dezember 2021).

Statista (2021b), Anteil der Jugendlichen und jungen Erwachsenen in Deutschland, der regelmäßig Alkohol konsumiert, im Zeitraum von 1973 bis 2019, https://de.statista.com/statistik/daten/studie/222971/ umfrage/alkoholkonsum-von-jugendlichen-und-jungen-erwachsenen-in-deutschland/ (15. Dezember 2021).

Tagesschau (2020), Gras vom Staat, https://www.tagesschau.de/ausland/amerika/hanf-uruguay-101.html (15. Dezember 2021).

Tagesschau (2021), Cannabis - „Made in Germany“, https://www.tagesschau.de/inland/innenpolitik/verkauf-cannabis-apotheken-arzneimittel-101.html (15. Dezember 2021).

Welt (2018), So würde Deutschland von einer Cannabis-Legalisierung profitieren, https://www.welt.de/wirtschaft/plus172367853/WieDeutschland-von-einer-Cannabis-Legalisierung-profitieren-wuerde. html (15. Dezember 2021).

Washington Post (2017), Following marijuana legalization, teen drug use is down in Colorado, https://www.washingtonpost.com/news/wonk/ wp/2017/12/11/following-marijuana-legalization-teen-drug-use-isdown-in-colorado/ (15. Dezember 2021).

WiWo (2015), Haschisch für alle, https://www.wiwo.de/politik/deutschland/plaedoyer-fuer-die-cannabis-freigabe-haschisch-fuer-alle/11617034.html (15. Dezember 2021).

Wissenschaftliche Dienste des Deutschen Bundestags (2019a), Sachstand: Strafrechtliche Aspekte des Umgangs mit Cannabis im internationalen Vergleich, WD 7-3000-134/19, https://www.bundestag.de/ resource/blob/665450/0925ea6a5994641b8b0bf340811b6fd3/WD-7134-19-pdf-data.pdf (15. Dezember 2021).

Wissenschaftliche Dienste des Deutschen Bundestages (2019b), Sachstand: Legalisierung von Cannabis Auswirkungen auf die Zahl der Konsumenten in ausgewählten Ländern, https://www.bundestag.de/ resource/blob/675688/4ba9aed6de8e9633685a1cdc2d823525/WD9-072-19-pdf-data.pdf.

Zeit (2016), Freund des Joints, https://www.zeit.de/2015/22/cannabisfreigabe-joachim-pfeiffer (15. Dezember 2021).

Title: Why and How the Liberalisation of the Cannabis Market in Germany Should Occur

Abstract: This article summarises the arguments in favour of legalising cannabis and makes proposals for the design of the regulatory framework. The main argument for a regulated cannabis market is not the expected tax revenues or the legal jobs that would be created, but the improved youth and health protection in a state-regulated cannabis market. While consumption of legal addictive substances such as tobacco and alcohol has been successfully curbed, cannabis consumption has been increasing for years despite prohibition. $A$ well designed market order with appropriate market regulation and taxation is crucial for effective youth and health protection. 